Synthesis of Natural

Products and

Potential Drugs

\section{Total Synthesis of (+)-Peganumine A}

\section{Key words}

\section{(+)-peganumine $A$}

Passerini reaction

Pictet-Spengler reaction

thiourea catalysis<smiles>COc1ccc2c(CCN)c[nH]c2c1</smiles>

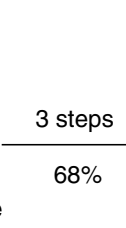

$\mathrm{N}^{\mathrm{O}}$<smiles></smiles>

B<smiles>O=P(O[Na])(c1ccccc1)c1ccccc1</smiles>

$$
\begin{aligned}
& \mathrm{D}, \mathrm{Ph}_{3} \mathrm{As}(10 \mathrm{~mol} \%) \\
& \text { hexane-THF }(3: 1)
\end{aligned}
$$<smiles>COc1ccc2c(CC[NH3+])c[nH]c2c1</smiles>

$\mathrm{NCS}, \mathrm{Me}_{2} \mathrm{~S}$
$\mathrm{CH}_{2} \mathrm{Cl}_{2},-78^{\circ} \mathrm{C}$

then $\mathrm{Et}_{3} \mathrm{~N},-78^{\circ} \mathrm{C}$

$\longleftarrow$

$96 \%$

Corey-Kim oxidation

G

\section{$4 \AA \mathrm{MS}, \mathrm{PhMe}, \triangle$ then I (20 mol\%)} $\mathrm{BzOH}(20 \mathrm{~mol} \%)$ $\mathrm{PhMe}-\mathrm{CH}_{2} \mathrm{Cl}_{2}(9: 1), 35^{\circ} \mathrm{C}$ then TFA (20 mol\%), $\Delta$
C, $\mathrm{Pd}_{2} \mathrm{dba}_{3}(10 \mathrm{~mol} \%)$ then $\mathrm{G},-78^{\circ} \mathrm{C}$
$\overbrace{}^{\mathrm{O}}$

$95 \%$

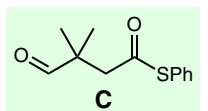

Liebeskind-Srogl cross-coupling<smiles>CCc1c(C(=O)CC(C)(C)C=O)[nH]c2cc(OC)ccc12</smiles>
$92 \% \quad \begin{gathered}\mathrm{POCl}_{3}, \mathrm{Et}_{3} \mathrm{~N} \\ \mathrm{CH}_{2} \mathrm{Cl}_{2},-78\end{gathered}$<smiles>C=CC(C)(C)CC(=O)c1[nH]c2cc(OC)ccc2c1CCNC#N</smiles>

A<smiles>CC(I)C(NC(=S)Nc1cc(C(F)(F)F)cc(C(F)(F)F)c1)C(=O)N(C)Cc1ccccc1</smiles><smiles>COc1ccc2c(CCN=C3C(=O)N4CCc5c([nH]c6cc(OC)ccc56)C4=CC3(C)I)c[nH]c2c1</smiles><smiles></smiles>

(+)-Peganumine A

Significance: (+)-Peganumine A, isolated from the seeds of Peganum harmala $L$., is a dimeric tetrahydro- $\beta$-carboline alkaloid displaying significant selective cytotoxic activity against HL-60 cells $\left(\mathrm{IC}_{50}=5.8 \mu \mathrm{M}\right)$. The first enantioselective synthesis by Zhu and co-workers relies on an early Liebeskind-Srogl cross-coupling and a thioureacatalyzed Pictet-Spengler reaction to form the unprecedented octacyclic scaffold.
Comment: Liebeskind-Srogl cross-coupling of stannane $\mathbf{B}$ and thioester $\mathbf{C}$ provided $\mathrm{N}$-formamide $\mathbf{E}$ in $95 \%$ yield. After dehydration, a three-centertwo-component Passerini reaction followed by oxidation furnished tetracycle $\mathbf{H}$. The synthesis was completed by an enantioselective Pictet-Spengler reaction of $\mathbf{H}$ and 6-methoxytryptamine $(\mathbf{A})$ to give (+)-peganumine A in a total of 7 steps and 33\% overall yield.

sYNFACTS Contributors: Erick M. Carreira, Marco Brandstätter

Synfacts 2016, 12(11), 1113 Published online: 18.10.2016

Dol: 10.1055/s-0036-1589311; Reg-No.: C06216SF 